\title{
Observation of interactions between two erupting filaments
}

\author{
Yu Liu ${ }^{1}$, Jiangtao $\mathrm{Su}^{2}$, Yuandeng Shen ${ }^{1}$ and Liheng Yang ${ }^{1}$ \\ ${ }^{1}$ National Astronomical Observatories, \\ Yunnan Astronomical Observatory, Kunming 650011, China \\ email: lyu@ynao.ac.cn \\ ${ }^{2}$ National Astronomical Observatories, Beijing 100012, China
}

\begin{abstract}
Following the first observational study of the interaction between two distinct filaments (Su et al. 2007; hereafter, event 1), we present another interesting case observed by SMART telescope on 2005 June 25 with higher spatial resolution (hereafter, event 2). The two events are compared with each other. In event 1 the two filaments erupted subsequently and obvious mass flow was observed to be transferred from one erupting filament to one stable filament which triggered its eruption. On the contrary, in event 2, the two filaments erupted simultaneously and there was no transfer of material noticed between them during the initial stage. The two filaments merged together along the ejection path, indicating the bodily coalesce between the two interacting flux ropes. Moreover, event 1 was associated with a coronal mass ejection $(\mathrm{CME})$, while event 2 was a failed filament eruption, thus without CME association.
\end{abstract}

Keywords. Sun: corona; Sun: magnetic fields; Sun: filament

\section{Introduction}

Generally, filament instabilities can be triggered by interior and exterior agents. The interior agents may be magnetic field flux evolution and cancelation on the photosphere. The exterior agents include fast running Moreton waves, remote extending flare ribbons and nearby repetitive surge injections (Liu et al. 2005, Jiang et al. 2009). Many previous studies have shown that filament disturbances are closely related to pore birth and movements which are results of magnetic flux emergence and cancelation. Moreover, the impact from an erupting filament can also be an exterior trigger for the eruption of a neighbor filament. Su et al. (2007) presented such a case well observed in three wavelengths of $\mathrm{H} \alpha$ line (1.e. event 1). In the case, both filaments were successfully erupted and accompanied by a CME. Fig. 1 shows the carton describing the process of two filament merge due to magnetic field reconnection between them. The reconnection can be directly caused by one filament impact onto another filament body. This model is consistent with many aspects of the $\mathrm{H} \alpha$ and EUV observations.

\section{New observations: event 2}

Two filament eruptions were observed on 2005 June 25 in the solar active region NOAA 10780 (S08,W30). They were associated with a C1.3-class flare (03:30-04:30 $\mathrm{UT}$ ), but there was no radio burst or coronal mass ejection reported for the event. The ground-based observations were taken by the SMART telescope at Hida Observatory. The SMART telescope was operated to take data at the $\mathrm{H} \alpha$ wavelength center and several off-bands, with a full disk mode and a local-monitoring mode in two observing channels. The time cadence was 7-30 s for most $\mathrm{H} \alpha$ data whose resolution was better 


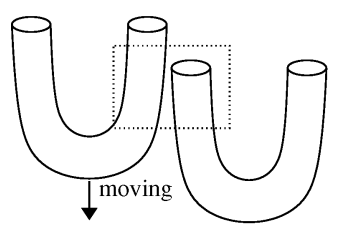

(a)

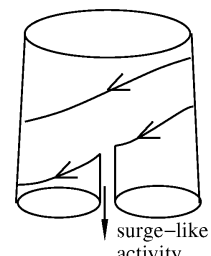

(c)

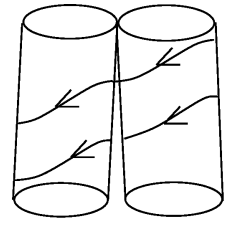

(b)

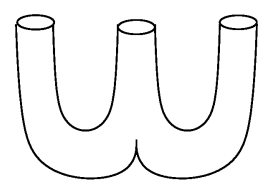

(d)

Figure 1. A schematic model showing the interaction and merge of two flux ropes located closely ( $\mathrm{Su}$ et al. 2007). The helical magnetic field components in the filaments are thought to play an important role during the interaction and merge process for two approaching flux ropes.

than 1 arcsec for good seeing conditions. We briefly show the $\mathrm{H} \alpha$ observations in Fig. 2 which presents the main development for the two filament eruptions as well as their morphology evolution. The time and the off-band information are supplied at the bottom for each frame. After the initial stage (Fig. $2 a-2 b$ ), the two filaments merged totally (Fig. $2 c-2 f)$.

\section{Conclusion}

$\mathrm{Su}$ et al. (2007) reported event 1 as the first filaments interaction and eruption event. In this paper we present event 2 as new observations. In event 1 , obvious mass flow was found to be injected from one erupting filament to another stable one, triggering its eruption subsequently. In event 2, however, filament merging process was observed soon after their interaction. The two filaments merged as one moving along the overlying coronal fields (Liu et al. 2009). In event 2, the north filament eruption was closely relevant to the underlying flux cancelation due to the collision of two magnetic fluxes with opposite polarity on the photosphere. The event was companied by a C1.3-class flare with two spreading ribbons, the EUV dimmings and the overlying post-flare arcades. Moreover, the event had no associated CME because it was a failed filament eruption and the filaments material returned back to solar surface. Single loop-like bright ejections were observed in Nobeyama radio flux data $(17 \mathrm{GHz})$, TRACE EUV and SXI SXR images. The event clear shows that the disturbing agent of a filament disturbance can be the impact from another filament, which is different from the other exterior agents, e.g., flare ribbons, surges and shock waves. The interaction and mergence between two filaments should involve complicated dynamic and thermal process. The detailed mechanism is beyond the scope of our current observations. We speculate that the interaction and mergence should experience a violent mass and magnetic field flux transfer and exchange. Numerical simulations are needed to reveal more details for understanding the interaction process.

Furthermore, both the filaments in event 2 were sinistral and thus their mergence was consistent with the fact found by Martin et al. (1994) that filaments or filament channels of same magnetic handedness may merge with each other while those of opposite handedness may not join. 


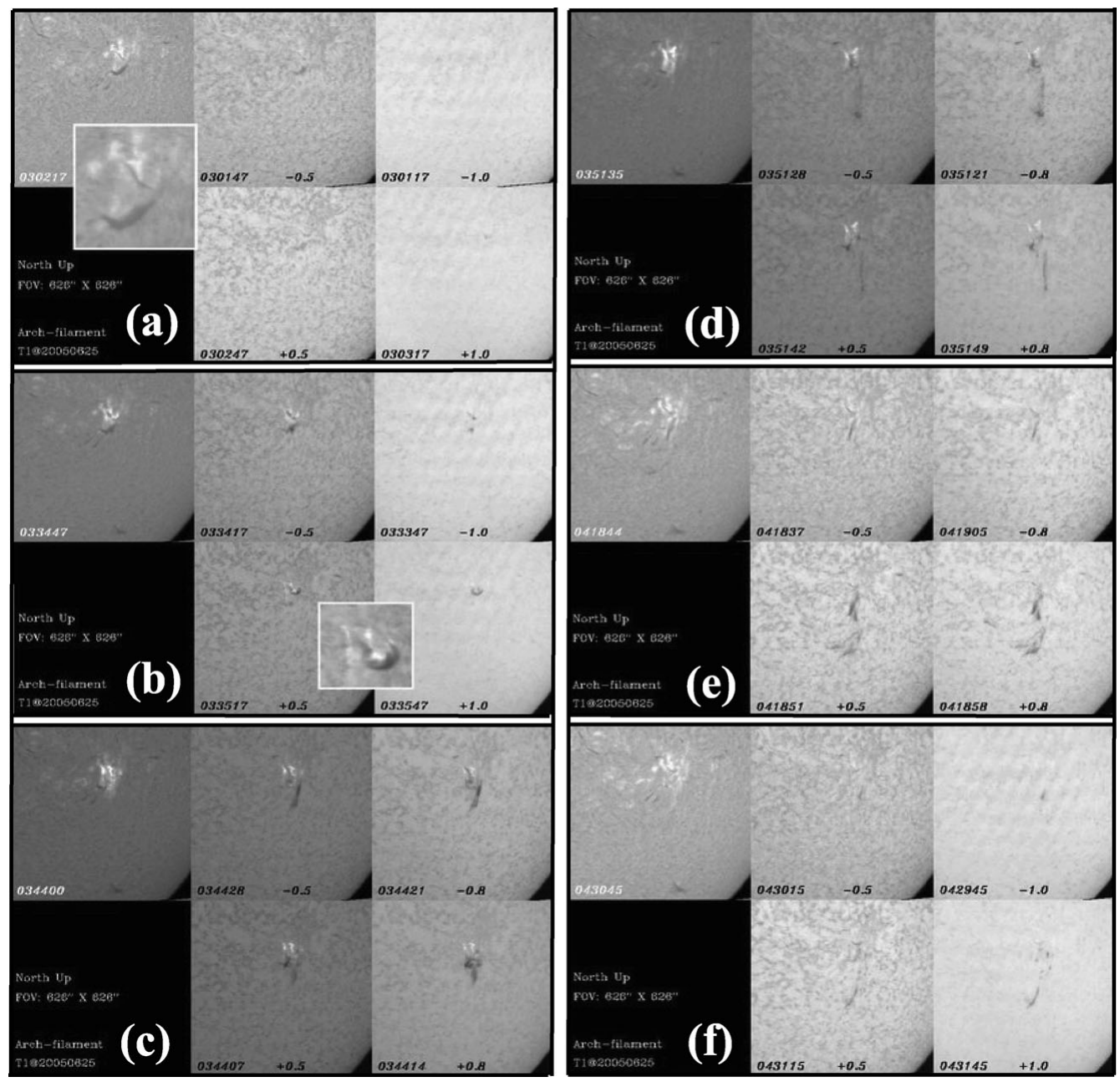

Figure 2. Multi-wavelength observations at $\mathrm{H} \alpha$ line for event 2 of two filaments interaction on 2005 June 25. (a) before eruption. (b) start of eruption and the north untwisting filament. $(c-d)$ filaments interaction during the eruption. $(e-f)$ filament material returned back to the surface and their eruptions were failed.

\section{Acknowledgements}

We are grateful to National Natural Science Foundation of China 10843010 and 10933003.

\section{References}

Jiang, Y., Bi, Y., Yang, J., Zheng, R., \& Wang, J. 2009, Research in Astro. \& A Astrophys., 9, 603 Martin, S., Billimoria, R., \& Tracadas, P. 1994, in: R. Rutten \& C. Schrijvers (eds.), Solar Surface Magnetism, (Kluwer Acad. Publ.), p. 303

Liu, Y., Kurokawa, H., \& Shibata, K. 2005, ApJ (Letters), 631, L93

Liu, Y., Su, J., Xu, Z., Lin, H., Shibata, K., \& Kurokawa, H. 2009, ApJ (Letters), 696, L70

Su, J., Liu, Y., Kurokawa, H., Mao, X., Yang, S., Zhang, H., \& Wang, H. 2007, Solar Phys., 242,53 